\title{
Comparison of Giemsa microscopy with nested PCR for the diagnosis of malaria in North Gondar, north-west Ethiopia
}

\author{
Abebe Alemu ${ }^{1}$, Hans-Peter Fuehrer ${ }^{2}$, Gebeyaw Getnet ${ }^{1 *}$, Afework Kassu ${ }^{3}$, Sisay Getie ${ }^{1}$ and Harald Noedl ${ }^{4}$
}

\begin{abstract}
Background: Malaria remains one of the leading communicable diseases in Ethiopia. Early diagnosis combined with prompt treatment is one of the main strategies for malaria prevention and control. Despite its limitation, Giemsa microscopy is still considered to be the gold standard for malaria diagnosis. This study aimed to compare the performance of Giemsa microscopy with nested polymerase chain reaction (nPCR) for the diagnosis of malaria in north-west Ethiopia.
\end{abstract}

Methods: A cross sectional study was conducted in public health facilities in North Gondar, from March 2013 to April 2013. A total number of 297 subjects with suspected malaria were enrolled in the study. Finger-prick blood samples were collected and examined for Plasmodium parasites using Giemsa microscopy and standard nPCR.

Results: Among the study participants, 61.6\% (183/297) patients tested positive for malaria by Giemsa microscopy of which, $72.1 \%$ (132/183) and 27.9\% (51/183) were diagnosed as Plasmodium falciparum and Plasmodium vivax, respectively. By $n P C R, 73.1 \%$ (217/297) were malaria-positive. Among microscopy-negative samples, 13.1\% (39/297) samples turned malaria-positive in nPCR. In nPCR, the rate of mixed Plasmodium infections was 4.7\% (14/297) and 3.03\% (9/297) were positive for Plasmodium ovale. Using nPCR as reference the sensitivity, specificity, positive predictive and negative predictive values of Giemsa microscopy were $82.0 \%, 93.8 \%, 97.3 \%$ and $65.8 \%$, respectively, with a good agreement $(k=0.668)$ to nested PCR. The sensitivity and specificity of Giemsa microscopy in identifying P. falciparium infections were $74.0 \%$ and $87.4 \%$ and $63.2 \%$ and $96.5 \%$ for $P$. vivax infections, respectively.

Conclusion: Although Giemsa microscopy remains the gold standard for malaria diagnosis in resource-limited environments, its sensitivity and specificity as compared to nPCR is limited suggesting exploration of novel rapid and simplified molecular techniques for malaria-endemic countries. A high rate of misclassification and misidentification highlights the importance of adequate training for staff involved in malaria diagnosis.

\section{Background}

Malaria is a disease caused by protozoans of the genus Plasmodium and continues to be one of the main causes of serious illness and death throughout the world. In spite of considerable improvements in some parts of the world, malaria-related morbidity and mortality have increased in other regions [1]. Malaria varies widely in epidemiology and clinical manifestations in different parts of the world. This variability is mainly due to differences

\footnotetext{
* Correspondence: ggebeyaw@yahoo.com

'Department of Medical Parasitology, School of Biomedical and Laboratory Sciences, College of Medicine and Health Sciences, University of Gondar, Gondar, Ethiopia

Full list of author information is available at the end of the article
}

in the species and prevalence of malaria parasites in different localities, difference in the susceptibility of Plasmodium to anti-malarial drugs, the distribution of mosquito vectors and immune status of the population [2].

Malaria is also among the leading communicable diseases in Ethiopia. It is estimated that 57.3 million (68\%) of the population of Ethiopia live in areas at risk of malaria [3]. The Federal Ministry of Health (FMOH) estimates that there are 5-10 million clinical malaria cases each year [4] accounting for $12 \%$ of outpatient consultations and $10 \%$ of admissions to hospitals [5]. In Ethiopia, Plasmodium falciparum and Plasmodium vivax are the main species accounting for approximately $60 \%$ and $40 \%$ 
of malaria cases, respectively [6] but changing epidemiology has resulted in a major shift from $P$. falciparum to $P$. vivax in the country [7]. Nonetheless, $P$. falciparum is the main causative of severe malaria in Ethiopia with case fatality rates of about $10 \%$ in hospitalized adults and up to $33 \%$ in children less than 12 years old [8].

Early and adequate diagnosis and prompt treatment is one of the main strategies in malaria prevention, control and effective disease management. In Ethiopia, different diagnostic methods are employed but diagnosis based on clinical signs and symptoms is still most frequently used in all peripheral areas where laboratory diagnosis is not available. However, clinical diagnosis is notoriously unreliable as the sign and symptoms of malaria are nonspecific and overlapping with those of other febrile diseases. In recent years the use of rapid diagnostic tests (RDTs) has gained major importance wherever laboratory diagnosis is not available (e.g. at health posts). However, microscopic diagnosis of malaria based on examination of Giemsa-stained blood films remains the gold standard in Ethiopia [9].

The sensitivity of Giemsa microscopic slide examination significantly varies across species and geographic localities. A study in Ethiopia reported a sensitivity that varied between $44 \%$ and $96 \%$, and a specificity of greater than $90 \%$ [10]. Microscopy offers significant advantages to other methods (like RDTs), but it has its own limitations, including: detection of low parasite loads, result interpretation, mixed infections and limited usefulness in non-endemic regions due to frequently inadequate training and experience of laboratory personnel [11].

An earlier study from north-west Ethiopia suggested that malaria microscopy leads to $16.3 \%$ false negative, $0.7 \%$ of false positive results and the misclassification of Plasmodium species [12]. The aim of this study was, therefore, to compare Giemsa microscopy to nested polymerase chain reactions (nPCR) for the diagnosis of malaria in North Gondar, north-west Ethiopia, to provide a better understanding of the reliability of Giemsa microscopy for malaria diagnosis in this region.

\section{Methods}

\section{Study area}

The study was conducted in a hospital (Metema hospital) and three health centres (Maksegnt, Enfranze and Kola Diba) in North Gondar areas which are known to be endemic for malaria. The altitude ranges from 1,750 to $2100 \mathrm{~m}$ above sea level. According to the Municipal Health Bureau report, malaria is the most prevalent seasonal disease in these areas.

\section{Study design, inclusion and exclusion criteria}

A diagnostic study was conducted from March 2013 to April 2013. Male and female febrile patients of any age suspected to be positive for malaria, willing to participate and to sign the informed consent were included in this study. Patients who had received anti-malarial drugs during the past four weeks and critically ill patients unable or unwilling to provide a blood sample were excluded from the study.

\section{Sample size and sampling techniques}

Study participants were recruited consecutively (convenience sampling) and a total of 297 patients with signs and symptoms consistent with malaria enrolled. Fingerprick blood samples were collected from every participant and placed on grease-free, clean microscopic glass slides. On a single slide, both thick and thin blood films were prepared. The thin blood films were fixed in methanol after air-drying before the slides were stained in a 30\% Giemsa solution for 10 minutes. Thin and thick blood films were read at the health centre by experienced medical laboratory technologists and the result was considered as negative if no Plasmodium parasites were seen after examining 100 high power (1000x) fields.

\section{Sample collection for molecular analysis}

Two blood spots were collected from each participant, transferred on filter paper (Whatman \#903, GE Healthcare) labelled with the participant's study code and date. Each filter paper was dried individually to avoid any chance of contamination. The samples were then stored in small plastic bags with desiccant and transported to the Institute of Specific Prophylaxis and Tropical Medicine, Medical University of Vienna (MUV), Vienna, Austria for molecular analysis.

\section{DNA isolation and parasite detection by nested PCR}

A modified chelex-based DNA extraction method using the InstaGene Whole Blood Kit (Bio-Rad Laboratories, Hercules, CA, USA) was used for the extraction and purification of Plasmodium DNA from the blood spots on filter paper. Parasite detection and species classification by nested PCR assay was performed for all samples as described previously $[13,14]$. The individual interpreting the PCR results was blinded to the results of microscopy.

\section{Data analysis}

Sensitivity, specificity and predictive values were determined using SISA online statistical software [15]. The kappa coefficient (Cohen's kappa coefficient as a measure of agreement for qualitative items) was determined to confirm the consistency of the results among the diagnostic tools. 


\section{Ethical consideration}

The study protocol had been reviewed and approved by the institutional review board (IRB) of the University of Gondar prior to the study. Written informed consent (consent and/or assent) was obtained from all participants or their legal guardians after being translated and read in the vernacular language. Patients testing positive for malaria by microscopy received immediate treatment according to the national treatment guidelines.

\section{Results}

Comparison of microscopy and nested polymerase chain reaction for the diagnosis of malaria and Plasmodium species identification in Northwest Ethiopia

A total of 297 febrile patients with suspected malaria based on clinical presentation were enrolled and screened for Plasmodium parasites in the course of the study. Among the study participants, 61.6\% (183/297) patients were malaria-positive by microscopy. Of those, $72.1 \%(132 / 297)$ and $27.9 \%$ (51/297) were diagnosed as $P$. falciparum and P. vivax, respectively, by Giemsa microscopy. Nested PCR was used as reference method and $73.1 \%(217 / 297)$ participants tested positive by nested PCR. Among microscopy-negative patients, 13.1\% (39/297) cases tested positive by nested PCR (Figure 1). In this study, Giemsa microscopy did not identify any mixed infections of Plasmodium parasites or any cases of Plasmodium ovale infections. However, $4.7 \%$ (14/297) samples were identified as mixed Plasmodium species infections by nested PCR and $P$. ovale was in $3.03 \%(9 / 297)$ patient samples (Table 1$)$.

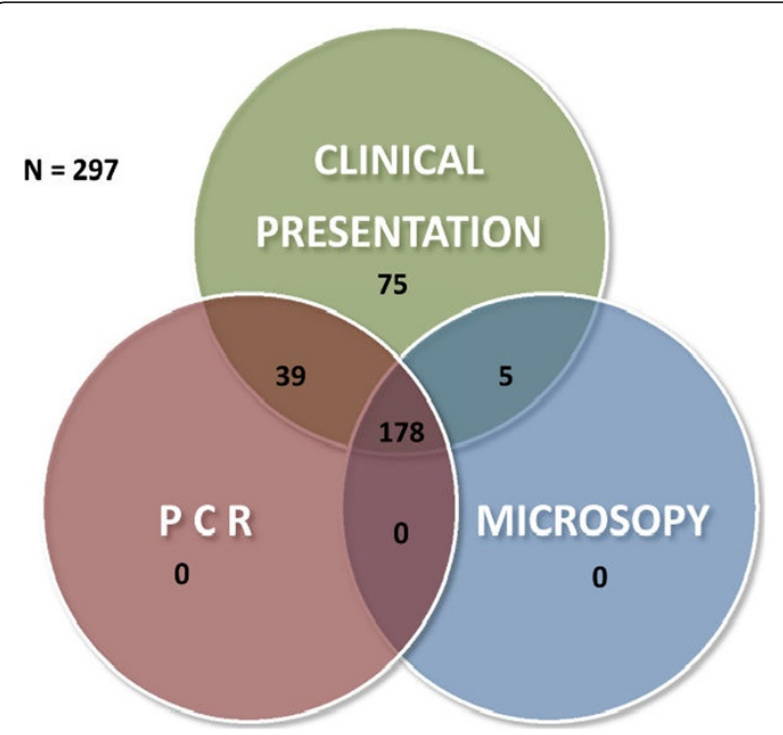

Figure 1 Venn diagram showing relationship between positivity in clinical presentation, Giemsa microscopy and $\mathrm{nPCR}$.
Table 1 Comparison of microscopy and nested polymerase chain reaction for malaria parasite detection and species identification in Northwest Ethiopia, 2013

Parasites detected by microscopy and nested PCR (Number of samples)

\begin{tabular}{|c|c|}
\hline Microscopy (n) & Nested PCR (n) \\
\hline $\begin{array}{l}\text { Plasmodium } \\
\text { falciparum (132) }\end{array}$ & $\begin{array}{l}\text { P. falciparum (108), P. vivax (11), P. ovale sp. (2), P. } \\
\text { falciparum + P. vivax (6), negative (5) }\end{array}$ \\
\hline $\begin{array}{l}\text { Plasmodium vivax } \\
(51)\end{array}$ & $\begin{array}{l}\text { P. vivax (38), P. falciparum (4), P. ovale sp. (2), P. vivax + } \\
\text { P. falciparum (5), P. falciparum + P. ovale sp. (2) }\end{array}$ \\
\hline Negative (114) & $\begin{array}{l}\text { Negative (75), P. falciparum (28), P. vivax (8), P. } \\
\text { ovale sp. (2), P. falciparum + P. ovale sp. (1) }\end{array}$ \\
\hline
\end{tabular}

$\mathrm{n}=$ number of blood samples examined.

Agreement, sensitivity, specificity and predictive values of Giemsa microscopy compared to nested PCR for Plasmodium detection and Plasmodium species identification

Giemsa microscopy showed a good measure of agreement $(\kappa=0.668)$ with the reference method, nested PCR for Plasmodium detection. Its sensitivity and specificity were $82.0 \%$ (95\% CI: 76.1-86.8) and 93.8\% (95\% CI: 85.497.7), respectively. The probability for a positive microscopy result to be true positive (positive predictive value, PPV) was 97.3\% (95\% CI: 93.4-99.0) and for a negative result to be true negative (negative predictive value, NPV) was 65.8\% (95\% CI: 56.2-74.3.1).

Taking mixed infections into account (a mixed infection counting for both species as positive) the $P$. falciparum-specific sensitivity of microscopy relative to nPCR was $74.0 \%$ (95\% CI: $66.2-80.6 \%$ ), the specificity $87.4 \%$ (95\% CI: $80.6-92.2 \%$ ), the positive and negative predictive values 86.4 (95\% CI: 79.0-91.5\%) and 75.8 (68.481.9\%), respectively. The corresponding values for $P$. vivax were $63.2 \%$ 95\% CI: (50.6-74.4\%) for sensitivity, 96.5\% (95\% CI: 93.0-98.4\%) for specificity, 84.3\% (95\% CI: 70.9-92.5\%) for PPV, and 89.8\% (95\% CI: 85.2-93.2\%) for NPV.

\section{Discussion}

Malaria is still a leading cause of morbidity in Ethiopia where $78 \%$ of the country and $68 \%$ of its population are at risk of malaria. Plasmodium falciparum contributes around $60-75 \%$ to the malaria burden and the only other species routinely identified being $P$. vivax [16,17]. As diagnostic resources are limited throughout Ethiopia, microscopy remains the laboratory standard for diagnosing malaria [18]. However, even under optimal conditions the sensitivity of microscopy is limited to approximately 20 parasites/ $\mu$ l of blood, and subjective interpretation and reader errors further reduce the accuracy of diagnosis [19]. Nested PCR has a somewhat lower limit of detection of approximately 6 parasites/ $\mu \mathrm{l}$ of blood when using dried blood sample [20,21] which 
depends on the sample used (e.g. dried versus fresh). In the current study, whole blood collected on filter paper has been used for nested PCR. The difference in sensitivity is obviously reflected in the different rates of positivity seen in this study (61.6\% by microscopy versus $73.1 \%$ in $\mathrm{nPCR}$ ).

Nested PCR utilizes genus and species specific markers for the detection of Plasmodium parasites. This allows for the detection of low density infections and even more importantly of mixed infections, which are routinely missed in microscopy [13] and makes nested PCR an ideal confirmatory test for malaria diagnosis. In the present study, 39 (13.1\%) sample considering negative by microscopy gave positive results in nested PCR.

These results are in concordance with those of other studies from Africa, Asia and Latin America reporting a considerably higher potential for detecting low parasite densities [22-26]. A recent study from Ethiopia suggests that PCR has a much higher potential for detecting subclinical infections [27].

In the present study, the sensitivity of Giemsa microscopy for the diagnosis of $P$. falciparum was considerably higher when compared to P. vivax (74.0\% vs. $63.2 \%)$, while at the same time the specificity was higher for $P$. vivax (87.4\% vs. 96.5\%) suggesting that microscopists tend to interpret slides that are difficult to read (either because of morphology, artefacts, or low parasitaemia) as $P$. falciparum rather than $P$. vivax.

Although Giemsa microscopy did not identify a single case of mixed infection, all $P$. vivax-P. falciparum infections were read as malaria-positive and approximately half of them were interpreted as $P$. falciparum monoinfections, the other half as $P$. vivax. This is somewhat surprising as previous experience shows that mixed infections tend to be interpreted as $P$. vivax rather than $P$. falciparum, obviously because of its characteristic morphology.

Until very recently, species other than $P$. falciparum and $P$. vivax had not been reported from the region [12]. This is in part due to the fact that training of microscopists largely focuses on the detection of the two most prevalent species but also on the fact that molecular data to confirm and/or augment microscopic results are rarely available. In this study, nine samples tested positive for $P$. ovale out of which six were monoinfections and three were mixed with $P$. falciparum. However, only three were interpreted as being negative by microscopy. Once again not a single sample tested positive for Plasmodium malariae.

In spite of the inherent limitations of malaria Giemsa microscopy, the quality of microscopic diagnosis largely depends on the quality of training [28]. On the one hand, adequate training can increase the yield of accurate malaria diagnosis which helps to reduce illness, potential death, mistreatment and persistently high disease burden while at the same time saving vital resources for malaria control [29,30].

The added cost of using molecular techniques as confirmatory diagnostic tool for the malaria control programs needs to be carefully balanced against the cost (not to mention the human cost) of a higher disease burden. In the near future PCR (or equivalently sensitive diagnostic tools) will not be available throughout most malaria-endemic regions but a network of reference centers could potentially support ongoing diagnostic and control efforts by malaria control programs on the long run even significantly reduce the overall cost by providing a more targeted approach to the challenges of the malaria eradication era.

\section{Competing interests}

The authors declare that they have no competing interests.

\section{Authors' contributions}

AA and HN conceived the study. AA and HFP undertook statistical analysis, undertook molecular analysis of the samples and drafted the manuscript. GG, SG and AK contributed in proposal writing, blood sample collection and microscopic diagnosis of malaria slides. All authors contributed to the writing of the manuscript and approved the submitted version of the manuscript.

\section{Acknowledgements}

We are grateful to MARIB (Malaria Research Initiative Bandarban) for funding this study. The authors wish to thank data collectors at each study site and the study participants for their cooperation in providing the necessary information and blood sample. Furthermore, we wish to thank Ingrid Bloschl for their excellent technical support.

\section{Author details}

'Department of Medical Parasitology, School of Biomedical and Laboratory Sciences, College of Medicine and Health Sciences, University of Gondar, Gondar, Ethiopia. ${ }^{2}$ Institute of Parasitology, Department of Pathobiology, University of Veterinary Medicine Vienna, Vienna, Austria. ${ }^{3}$ Department of Medical Microbiology, School of Biomedical and Laboratory Sciences, College of Medicine and Health Sciences, University of Gondar, Gondar, Ethiopia. ${ }^{4}$ Institute of Specific Prophylaxis and Tropical Medicine, Medical University of Vienna, Vienna, Austria.

Received: 16 February 2014 Accepted: 2 May 2014

Published: 7 May 2014

\section{References}

1. Rosenthal PH: Antimalarial Chemotherapy. Mechanism of action, resistance, and new directions in drug discovery. Totowa, New Jersey: Humana press; 2001:65-87.

2. Bloland PB: Drug Resistance in Malaria. Available at http://www.who.int/emc (accessed on May 25, 2012

3. President's malaria initiative: Malaria Operational Plan FY 2013. Ethiopia: President's malaria initiative; 2013

4. President's malaria initiative: Malaria Operational Plan FY 2012. Ethiopia: President's malaria initiative; 2012

5. President's malaria initiative: Malaria Operational Plan FY 2011. Ethiopia: President's malaria initiative; 2011

6. Adugna A: Malaria in Ethiopia. Available at www. ethioDemographyAndhealth.org [accessed on December 10, 2012].

7. Alemu A, Tesfaye W, Golassa L, Abebe G: Climatic variables and malaria transmission dynamics in Jimma town, South West Ethiopia. Parasit Vectors 2011, 4:30.

8. Mitikie G, Addissie M, Admassu M, Tadesse A, Feleke A, Alebachew H, Malaria For the Ethiopian Health Center Team: Gondar College of Medicine and Health Sciences. In Collaboration with the Ethiopia Public Health 
Training Initiative, the Carter Center, the Ethiopia Ministry of Health and the Ethiopia Ministry of Education, 2005:17.

9. Federal Ministry of Health of Ethiopia: Malaria Diagnosis and Treatment a Guideline for Health Workers in Ethiopia. Addis Ababa, Ethiopia: Federal Ministry of Health; 2004.

10. Endeshaw T, Graves PM, Ayele B, Mosher AW, Gebre T: Performance of local light microscopy and the ParaScreen Pan/Pf rapid diagnostic test to detect malaria in Health Centers in Northwest Ethiopia. PLoS One 2012, 7:4.

11. Wilson ML: Laboratory diagnosis of malaria (Conventional and Rapid Diagnostic Methods). Arch Pathol Lab Med 2013, 137:805-811.

12. Alemu A, Fuehrer HP, Getnet G, Tessema B, Noedl H: Plasmodium ovale curtisi and Plasmodium ovale wallikeri in North-West Ethiopia. Malar J 2013, 12:346.

13. Singh B, Bobogare A, Cox-Singh J, Snounou G, Abdullah MS, Rahman HA: A genus- and species-specific nested polymerase chain reaction malaria detection assay for epidemiologic studies. J Trop Med Hyg 1999, 60:687-692.

14. Fuehrer HP, Stadler MT, Buczolich K, Bloeschl I, Noedl H: Two techniques to simultaneously identify Plasmodium ovale curtisi and $P$. ovale wallikeri using the small subunit rRNA gene. J Clin Microbiol 2012, 50:4100-4102.

15. Uitenbroek DG: SISA-Binomial. 1997. http://www.quantitativeskills.com/sisa/ distributions/binomial.htm. (1 Jan. 2014).

16. World Health Organization: World Malaria Report 2012. Geneva: WHO; 2012

17. Alemu A, Muluye D, Mihret M, Adugna M, Gebeyaw M: Ten year trend analysis of malaria prevalence in Kola Diba, North Gondar, Northwest Ethiopia. Parasit Vectors 2012, 5:173.

18. Federal Ministry of Health: National Malaria Guidelines. 3rd edition. Addis Ababa, Ethiopia: federal ministry of health; 2012.

19. Royal Tropical Institute: Malaria Diagnosis. KIT Biomedical Research Diagnostics development. Available at http://www.kit.nl/kit/malariadiagnostics (accessed on December 11, 2012.

20. Patsoula E, Spanakos G, Sofianatou D, Parara M, Vakalis NC: A single-step, PCR-based method for the detection and differentiation of Plasmodium vivax and P. falciparum. Am J Trop Med Hyg 2003, 97:15-22.

21. Fuehrer HP, Noedl H: Recent advances in the diagnosis of Plasmodium ovale. Implications of the separation into the two species $P$. o. wallikeri and P. o. Curtisi for diagnosis. J Clin Microbiol 2013, 5:20-32.

22. Abdel-Wahab MM, Ismail KA, El-Sayed NM: Laboratory diagnosis of malaria infection in clinically suspected cases using microscopic examination, optimal rapid antigen test and PCR. Parasitol United J 2012, 5:59-56.

23. Wangai LN, Karau MG, Njiruh PN, Sabah O, Kimani FT, Magoma G, Kiambo $\mathrm{N}$ : Sensitivity of microscopy compared to molecular diagnosis of $P$. falciparum: implications on malaria treatment in epidemic areas in Kenya. Afr J Infect Dis 2011, 5:1-6.

24. Rodulfo H, De Donato M, Mora R, González L, Contreras CE: Comparison of the diagnosis of malaria by microscopy, immunochromatography and PCR in endemic areas of Venezuela. Braz J Med Biol Res 2007, 40:535-543.

25. Zakeri S, Najafabadi ST, Zare A, Djadid ND: Detection of malaria parasites by nested PCR in south-eastern Iran: Evidence of highly mixed infections in Chahbahar district. Malar J 2002, 1:2.

26. Paris DH, Imwong M, Faiz AM, Hasan M, Yunus EB, Silamut K, Lee SJ, Day NP, Dondorp AM: Loop-mediated isothermal PCR (LAMP) for the diagnosis of falciparum malaria. Am J Trop Med Hyg 2007, 77:972-976.

27. Golassa L, Enweji N, Erko B, Aseffa A: Detection of a substantial number of sub-microscopic Plasmodium falciparum infections by polymerase chain reaction: a potential threat to malaria control and diagnosis in Ethiopia. Malar J 2013, 12:352.

28. Ministry of Health: Malaria Microscopy Competency in Liberia: Improving Malaria Diagnostics, 2010.

29. Foundation for Innovative New Diagnostics: FIND Malaria Strategy, 2012

30. Zurovac D, Larson BA, Akhwale W, Snow RW: The financial and clinical implications of adult malaria diagnosis using microscopy in Kenya. Trop Med Int Heath 2006, 11:1185-1194.

doi:10.1186/1475-2875-13-174

Cite this article as: Alemu et al:: Comparison of Giemsa microscopy with nested PCR for the diagnosis of malaria in North Gondar, north-west Ethiopia. Malaria Journal 2014 13:174.

\section{Submit your next manuscript to BioMed Central and take full advantage of:}

- Convenient online submission

- Thorough peer review

- No space constraints or color figure charges

- Immediate publication on acceptance

- Inclusion in PubMed, CAS, Scopus and Google Scholar

- Research which is freely available for redistribution

Submit your manuscript at www.biomedcentral.com/submit
C) Biomed Central 